\title{
Angular restriction fluorescence optical projection tomography to localize micrometastases in lymph nodes
}

\author{
Veronica C. Torres \\ Chengyue Li \\ Yusheng He \\ Lagnojita Sinha \\ Georgia Papavasiliou \\ Husain A. Sattar \\ Jovan G. Brankov \\ Kenneth M. Tichauer
}




\section{Angular restriction fluorescence optical projection tomography to localize micrometastases in lymph nodes}

\author{
Veronica C. Torres, ${ }^{a}$ Chengyue $\mathrm{Li}^{\mathrm{a}}{ }^{\mathrm{a}}$ Yusheng He,${ }^{\mathrm{a}}$ \\ Lagnojita Sinha, ${ }^{a}$ Georgia Papavasiliou, ${ }^{a}$ \\ Husain A. Sattar, ${ }^{b}$ Jovan G. Brankov, ${ }^{a, c}$ and \\ Kenneth M. Tichauer ${ }^{a, *}$ \\ allinois Institute of Technology, Department of Biomedical \\ Engineering, Chicago, Illinois, United States \\ bUniversity of Chicago, Department of Pathology, Chicago, \\ Illinois, United States \\ clllinois Institute of Technology, Department of Electrical and \\ Computer Engineering, Chicago, Illinois, United States
}

\begin{abstract}
Lymph node biopsy is a primary means of staging breast cancer, yet standard pathological techniques are time-consuming and typically sample less than $1 \%$ of the total node volume. A low-cost fluorescence optical projection tomography (OPT) protocol is demonstrated for rapid imaging of whole lymph nodes in three dimensions. The relatively low scattering properties of lymph node tissue can be leveraged to significantly improve spatial resolution of lymph node OPT by employing angular restriction of photon detection. It is demonstrated through porcine lymph node metastases models that simple filtered-backprojection reconstruction is sufficient to detect and localize $200-\mu \mathrm{m}$-diameter metastases (the smallest clinically significant) in 1-cm-diameter lymph nodes. ( $)$ The Authors. Published by SPIE under a Creative Commons Attribution 4.0 Unported License. Distribution or reproduction of this work in whole or in part requires full attribution of the original publication, including its DOI. [DOI: 10 .1117/1.JBO.24.11.110501]
\end{abstract}

Keywords: optical projection tomography; fluorescence; angular domain; lymph node; metastases; mesoscopic.

Paper 190260LR received Jul. 23, 2019; accepted for publication Oct. 14, 2019; published online Nov. 8, 2019.

Timely and accurate diagnoses of metastatic cancer have proven to be the single greatest influencer of positive therapeutic outcomes, improving 5-year survival rates by more than $10 \%$; thus, more sensitive methods for detecting cancer spread are of significant clinical interest. ${ }^{1,2}$ The current standard for identifying metastases in breast cancer (and a number of other cancers) is through pathological assessment of sentinel lymph node biopsy (SLNB) tissues. Typically, excised lymph nodes located during tumor resection surgery are cut to 2-mm-thick sections, processed, and stained with hemotoxylin and eosin for pathology reading (total process takes $24 \mathrm{~h}$ with pathologist read time of 1 to $2 \mathrm{~min})^{3,4}$ While this yields an analysis of only $1 \%$ of the lymph node volume, there is evidence that only macrometastases (cancer cell clusters of greater than $2 \mathrm{~mm}$ in diameter) have clinical

*Address all correspondence to Kenneth M. Tichauer, E-mail: ktichaue@iit.edu relevance. ${ }^{1,5,6}$ Yet, other works have indicated that micrometastases $\left(0.2\right.$ to $2 \mathrm{~mm}$ in diameter) also have clinical relevance. ${ }^{7,8}$ For more rapid assessments, frozen section pathology has become widely adopted, ${ }^{4}$ yet sensitivity and specificity have been reported to be variable from institute to institute, ${ }^{4,6}$ and $2-\mathrm{mm}$ node sectioning only ensures detection of macrometastases. Micrometastasis detection could be ensured by sectioning nodes at $0.2 \mathrm{~mm}$; however, this would make pathologist reading time go from 1 to $2 \mathrm{~min}$ to 10 to $20 \mathrm{~min}$. A more accurate and rapid method to detect both micro- and macrometastases could help overcome these challenges.

Optical projection tomography (OPT) offers a potential solution for rapid, whole sample evaluation. As demonstrated by Kokolakis et al., ${ }^{9}$ optical computed tomography was used to successfully distinguish between malignant and benign lesions in skin biopsies, as well as the extent of invasion, prior to histology through evaluation of reconstructed total attenuation coefficient maps. OPT along with fluorescence (emission-OPT) has been used extensively to provide three-dimensional (3-D) visualization of mesoscopic-sized samples with microscopic resolution. For instance, imaging of individual islets, microvasculature, and gene expression from whole rodent organs, embryos, and xenograft tumors were achieved; ${ }^{10-12}$ fluorescence lifetime imaging with OPT enabled functional imaging of biological samples, ${ }^{13,14}$ and to push the limits of resolution further, methods of light sheet microscopy, such as selective plane illumination microscopy, have been combined with OPT. ${ }^{15-17}$ Such techniques have even been used in ex vivo lymph node samples to visualize vascular networks and quantify cellularity. ${ }^{17,18}$ All of these works, however, require the sample to be optically cleared, a process which is both time and labor intensive. ${ }^{19}$ To combat this, methods of mesoscopic fluorescence tomography make use of mathematical models of photon propagation or other computational techniques to permit imaging of nontransparent samples. ${ }^{20-22}$ While these have demonstrated an ability to achieve necessary levels of spatial resolution, performance relies heavily on accurate modeling that can make image reconstruction computationally expensive. Alternatively, scatter rejection methods in the time ${ }^{23,24}$ and angular domains ${ }^{25}$ yield lower photon budgets but have the potential to be effective with very low-cost instrumentation and simple filtered-backprojection (FBP) for image reconstruction. In time-domain rejection, only the earliest arriving photons, through optical or electronic gating, are measured. On the other hand, in angular-domain rejection, only the straightest traveling photons, by restricting the detector's acceptance angle, are measured. Since there is a trade-off between the level of scatter rejection and measured number of photons, the amount of spatial resolution enhancement and manner in which it is achieved (time or angle restriction) depends on the application. Specifically, for objects with the size and optical properties of lymph nodes, it has been demonstrated, in absorption-based OPT simulation study, that angular restriction with a numerical aperture (NA) $=0.005$ is sufficient to detect and localize 0.2mm-diameter objects. ${ }^{26}$ While small NA is common in standard OPT to maximize depth of field, the level required for scatter rejection as demonstrated in the aforementioned study is an order of magnitude lower than what is typically used. Using the time-resolved lymph node transmittance optical property measurements and OPT system optimization (e.g., NA and associated optical components) with Monte Carlo simulation in the work done by Sinha et al., ${ }^{26}$ a fluorescence-based simulation 
study was conducted where it was demonstrated that up to $100 \%$ detectability and $95 \%$ localization of $0.2-\mathrm{mm}$ micrometastases in 10-mm-diameter nodes could be achieved with FBP reconstruction. ${ }^{27}$ These predictions were generated from a system configuration with expanded beam illumination, no time restriction, a detection $\mathrm{NA}=0.005$, and clinically feasible levels of fluorescence contrast $(100 \mathrm{nM})$; therefore, verifying the potential for implementation of a simple, low-cost, and relatively quick system with the ability to detect and localize the smallest clinically significant metastases. The work presented here capitalizes on this finding for the development of a lymph node imaging protocol using angular restriction fluorescence OPT. In conjunction with this, we have been developing paired-agent strategies for microscopic sensitivity to cancer cells in lymph nodes both in vivo ${ }^{28}$ and ex vivo; however, its details are outside the scope of this paper.

To demonstrate the advantage of scatter rejection in the angular domain for lymph node applications, fluorescence imaging was done on various porcine tissues using no restriction $(\mathrm{NA}=0.1333)$ and strict angular restriction $(\mathrm{NA}=0.005)$. Approximately 0.2-mm-diameter fluorescent agarose inclusions composed of IRDye $800 \mathrm{CW}$ (LI-COR Biosciences) were embedded as accurately as possible in the center of similar sized ( $\sim 1$-cm thick) lymph node, muscle, and fat tissues. The samples were suspended in transparent $1 \%$ agarose gel and placed on a motorized rotation stage for imaging on the in-house made angular restricted OPT system [schematic shown in Fig. 1(a)]. A 780-nm laser (FPL-02RFF1 Calmar Laser, Medocino, Palo Alto, California) was passed through a 10-nm-bandpass excitation filter (Chroma Technology, Rockingham, Vermont) and expanded using a Keplerian lens system [25- and 300-mm focal length lens (all lenses from ThorLabs, Newton, New Jersey)] to
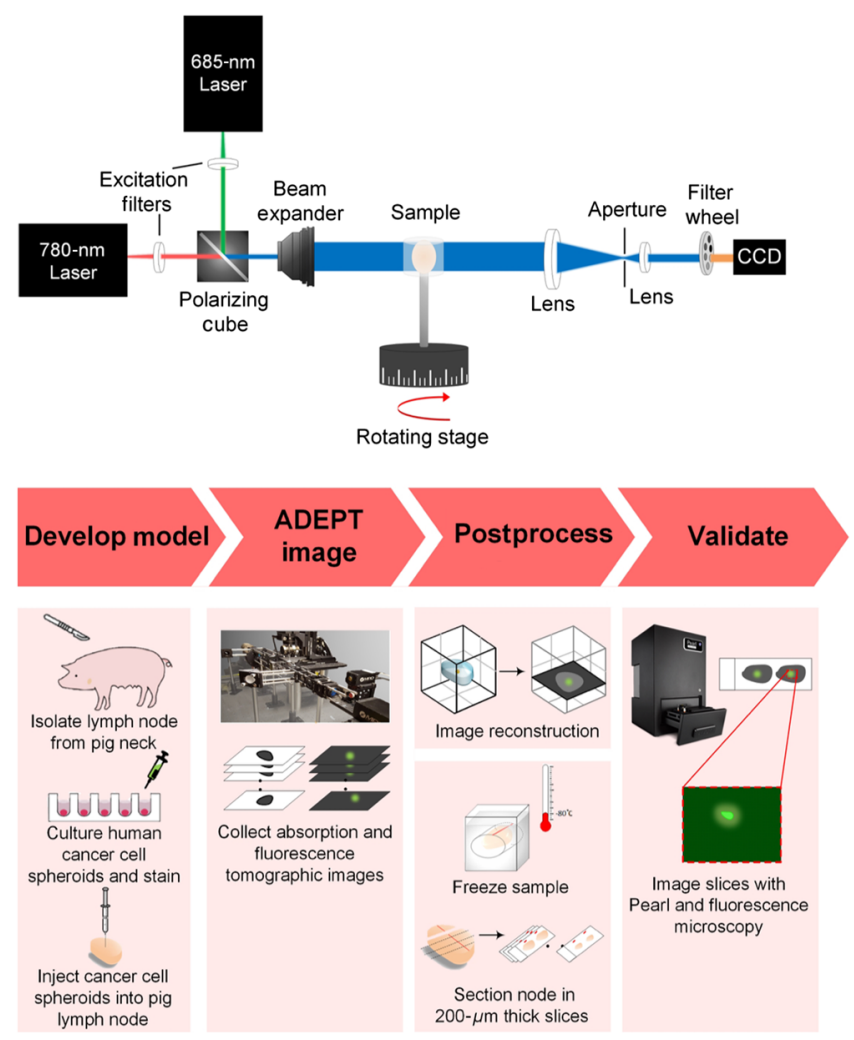

Fig. 1 (a) System schematic. (b) Experimental protocol. a beam waist of $2.4 \mathrm{~cm}$, to illuminate the whole surface of the tissue from one direction. Fluorescent light exiting the sample was collected directly opposite the illumination using a telecentric lens system (100- to 25-mm focal length lens) to focus down the light through an aperture before reaching the camera (sCMOS; Quantalux, ThorLabs). A continuously variable iris diaphragm (CP20S, ThorLabs) served as an aperture to restrict detection NA and was positioned between the lenses, at the focal length of each yielding an $\mathrm{NA}=0.133$ for no restriction or NA $=0.005$ for strict restriction. Emission light was filtered using a 45-nm notch filter centered at $780 \mathrm{~nm}$ (Chroma Technology). Camera exposure time was set to $1 \mathrm{~s}$ for images with no restriction and $5 \mathrm{~s}$ when strict restriction was implemented. All raw fluorescence signals in each setup were scaled independently and thresholded for visualization purposes such that $90 \%$ of the fluorescence range above the background is shown.

Results for each of the different tissue types using no angular restriction and strict angular restriction are presented in Fig. 2. It can be seen that with a completely open iris diaphragm, the fluorescent signal was more diffuse than when scatter rejection was employed with a closed iris. Figures 2(c), 2(f), and 2(i) compare fluorescence intensity line profile plots for each system configuration (no versus strict angular restrictions) in lymph node tissue, fat tissue, and muscle tissue, respectively. Profiles were calculated as the average of 100 rows at $0 \mathrm{deg}, 45 \mathrm{deg}$, $90 \mathrm{deg}$, and $125 \mathrm{deg}$ across each image, for a total of four measurements (only the 0-deg profiles are plotted in Fig. 2); mean values of full width at half maximum (FWHM) \pm standard deviation are summarized in Table 1. Lymph node tissue presented the greatest decrease (1.6×) in FWHM when going from NA $=0.133$ to 0.005 , muscle tissue was least impacted (1.2X decrease), and fat fell between the two, with a $1.4 \times$

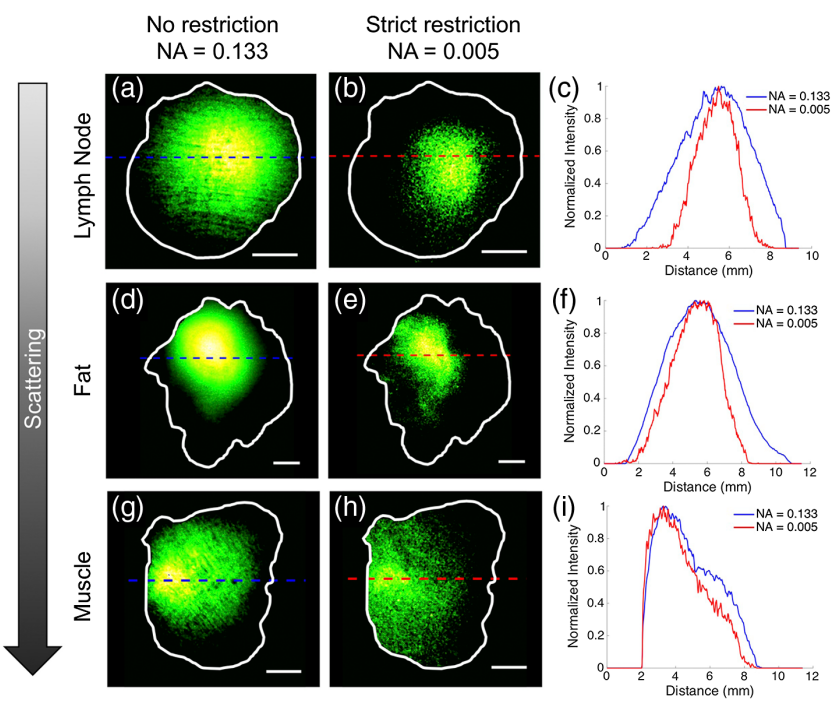

Fig. 2 Porcine tissues (top row, lymph node; middle row, fat; and bottom row, muscle) embedded with a fluorescent inclusion. Tissues are ordered to represent expected levels of optical scattering increasing from top to bottom. Columns display: false-colored fluorescence images from a single tomographic view using (a), (d), (g) no angular restriction (NA =0.133); (b), (e), (h) strict angular restriction (NA = 0.005). (c), (f), (i) Corresponding line profiles of normalized signal intensity. Tissue structures are outlined in white and scale bars are all $2 \mathrm{~mm}$. Dashed lines indicate the center of 100 averaged rows of the intensity profiles plotted for zero and strict constriction in blue and red, respectively. 
Table 1 Values of FWHM of fluorescence intensity profiles for tissues with a fluorescent inclusion.

FHWM (mm)

\begin{tabular}{lll} 
Tissue & $\mathrm{NA}=0.133$ & $\mathrm{NA}=0.005$ \\
\hline Lymph node & $4.21 \pm 0.16$ & $2.67 \pm 0.22$ \\
Fat & $5.02 \pm 0.45$ & $3.78 \pm 1.70$ \\
Muscle & $3.79 \pm 0.67$ & $3.22 \pm 0.50$ \\
\hline
\end{tabular}

improvement. The results were consistent with what was expected based on tissue optical properties, namely scattering; where compared to soft tissue-like muscle and fat, lymph nodes are much lower scattering in nature $\left(\mu_{s}=43 \mathrm{~cm}^{-1}, g=0.92\right.$, $\mu_{s}^{\prime}=3.37 \mathrm{~cm}^{-1}$ at $\left.780 \mathrm{~nm}^{26}\right)$. Reported values for piglet muscle are $\mu_{s}=239 \mathrm{~cm}^{-1}, g=0.732, \mu_{s}^{\prime}=62.1 \mathrm{~cm}^{-1}$ and $\mu_{s}=179 \mathrm{~cm}^{-1}, g=0.858, \mu_{s}^{\prime}=24.7 \mathrm{~cm}^{-1}$ measured at 630 and $632.8 \mathrm{~nm}$, respectively. ${ }^{29}$ Human subcutaneous adipose tissue, meanwhile, had reduced scattering coefficients between 11.3 and $21.1 \mathrm{~cm}^{-1}$ at $780 \mathrm{~nm} .{ }^{29}$ The anisotropy factor was not provided; however, using the average value for biological tissue $(g=0.9)$, an estimated average of $\mu_{s}=162 \mathrm{~cm}^{-1}$ could be deduced. Overall, an inverse relationship was found between scattering properties and resolution improvement with angular restriction; that is, as scattering increased, the difference in FWHM with and without angular restriction decreased. Muscle tissue revealed relatively similar results with and without scatter rejection, which can be attributed to its high "scattering power"- a parameter used to characterize the reduced scattering coefficient, $\mu_{s}^{\prime}$, in Jacques' in-depth review of biological tissue optical properties. ${ }^{30}$ In fact, muscle had reported a 2.7 times greater scattering power than fatty tissue. Fat, which had intermediate scattering properties, therefore showed improvements between that of lymph nodes and muscle. These findings support the conclusions of the previous simulation study ${ }^{26}$ in that angle restriction is an appropriate scatter rejection method specifically for lymph node applications because of its mesoscopic size and low scattering optical properties. While the resolution is obviously not sufficient to localize the $200-\mu \mathrm{m}$ inclusions from these single projections, based on the aforementioned simulation, ${ }^{27}$ it is expected that, upon tomography and reconstruction, they would be detected and localized with ease.

To test this, experiments were conducted with a porcine lymph node metastases model [Fig. 1(b)]. A human breast cancer cell line (MDA-MB-231) with known elevated expression of epidermal growth factor receptor (EGFR) was transfected with green fluorescent protein (GFP) and grown in 3-D cell culture so that cells could form spheroids to mimic the architecture of metastases. The spheroids were allowed to grow to a diameter of $\sim 200 \mu \mathrm{m}$. They were then stained for $60 \mathrm{~min}$ with a 100-nM solution of targeted fluorescent dye [IRDye 800DXlabeled cetuximab (an anti-EGFR antibody)] and rinsed with phosphate-buffered saline for $5 \mathrm{~min}$ before being implanted in two spots of lymph nodes excised from fresh porcine neck tissue acquired from a local butcher $(n=7)$. Inclusions were placed more superficially for whole node wide-field fluorescence imaging (Pearl, LI-COR, Lincoln, Nebraska) to verify successful implantation.

The same imaging procedure described above was carried out, except only strict restriction was applied $(\mathrm{NA}=0.005)$ and tomography was employed by capturing images in 5-deg intervals over $360 \mathrm{deg}$ for a total of 72 tomographic views. The same collection range was applied for absorption images (no fluorescence filter). Reconstruction was done on both (fluorescence and absorption) data sets using FBP to create 3-D fluorescence maps of the micromets and to recreate the node volume for structural context. Two-dimensional (2-D) slices at the height of detected cells were also reconstructed. Following imaging, the samples were frozen-sectioned in OCT at $200-\mu \mathrm{m}$-thick slices and imaged on a commercial wide-field fluorescence imaging system for comparison to reconstructed slices (Pearl Imager, LI-COR Biosciences) and on a fluorescent microscope (Axiovert, Zeiss) to validate the presence of cancer cells.

Results of two representative lymph nodes shown in Fig. 3 demonstrate the ability of the in-house developed system to detect and localize 0.2-mm-diameter micrometastases in $\sim 1$-cmdiameter lymph nodes. The absorption images with overlaid fluorescence from a single tomographic view provided visualization of the two micrometastases in each of the nodes [Figs. 3(a) and 3(h)]; and 2-D FBP reconstructed slices at the heights of where the fluorescent hot spots were revealed in whole node images verified their presence [Figs. 3(b), 3(e), 3(i), and 3(1)]. This was confirmed in both the fluorescent wide-field [Figs. 3(c), 3(f), 3(j), and 3(m)] and microscopy images [Figs. 3(d), 3(g), 3(k), and 3(n)] of physically sliced sections matching those that were reconstructed. Since both micrometastases were detected for all samples (and with relative ease), the predicted $100 \%$ detectability from simulation was supported. More rigorous experimental validation through quantitative performance metrics will be applied in future work; however, the qualitative analysis presented here suggests positive localization capabilities. The fluorescence microscopy
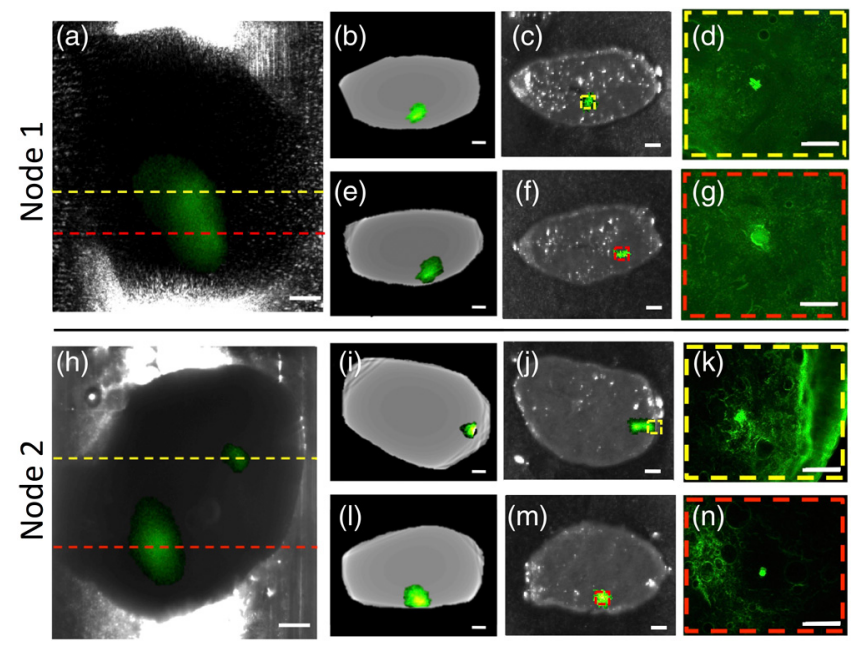

Fig. 3 Porcine lymph nodes implanted with GFP-labeled human breast cancer cell (MDA-MB-231) spheroids. Columns from left to right: (a), (h) false-colored fluorescence overlaid onto transmittance images from a single tomographic view (scale bar $1 \mathrm{~mm}$ ); (b), (e), (i), (l) angle-restricted fluorescence OPT FBP reconstructed virtual sections at the height of detected cells indicated by yellow and red dashed lines (scale bar $1 \mathrm{~mm}$ ); (c), (f), (j), (m) Pearl images (fluorescence overlaid on to white light) of lymph node sections sliced at the same heights (scale bar $1 \mathrm{~mm}$ ); (d), (g), (k), (n) fluorescent microscope images of the regions outlined in dashed boxes (scale bar $200 \mu \mathrm{m}$ ). Top and bottom rows for each node correspond to top (yellow dashed lines) and bottom (red dashed lines) detected micrometastases, respectively. 
images showed that the measured fluorescent signal was indeed from the GFP-labeled cancer cells. Note that background signal in the microscopy images can be attributed to autofluorescence in the GFP channel (517-nm emission peak; $525 \pm 25$-nm filter set). Higher intensity spots, as shown in the top microscopy image of node 2 [Fig. 3(k)], can be found near the edge of the sample because of stronger autofluorescence of collagen (420- to 510-nm emission), ${ }^{31}$ which makes up the fibrous capsule surrounding the node.

In this letter, preliminary results that support the development of a low-cost angular-domain imaging system to enhance the sensitivity of SLNB pathology were presented. Through porcine lymph node metastases models, simulation-predicted levels of detectability and localization of the smallest clinically relevant metastases were recapitulated using simple angular restriction and FBP reconstruction techniques. Ultimately, this demonstrates the potential for such a system and protocol to outperform conventional pathology by providing 3-D maps of cancer cell spread, which can eliminate blind gross-sectioning and in turn reduce the high rate of false negatives in breast cancer diagnosis. Future steps will include the use of task-based evaluation metrics to compare performance of the developed angular restriction fluorescence OPT system to current standard methods; the investigation of iterative reconstruction techniques for improved image quality; and implementation of a pairedagent staining protocol to further enhance cell detection. In addition, intermediate degrees of angular restriction, increased light source powers (a 2-order-of-magnitude increase from this work will remain below the ANSI safety limit), and noncoherent light sources will be evaluated in future to minimize imaging times, while maintaining an adequate level of signal-to-noise for accurately carrying-out the desired task of the system (e.g., micrometastasis localization).

\section{Disclosures}

The authors have no financial or other potential conflicts of interest to disclose.

\section{Acknowledgments}

The authors would like to acknowledge the financial support provided by the Pritzker Fellowship in Biomedical Sciences and Engineering at Illinois Institute of Technology. The research was supported by Nayar Prize I at Illinois Institute of Technology and grants from the U.S. National Science Foundation (CAREER 1653267) and U.S. National Institutes of Health (R01 EB023969).

\section{References}

1. M. de Boer et al., "Micrometastases or isolated tumor cells and the outcome of breast cancer," N. Engl. J. Med. 361(7), 653-663 (2009).

2. M. A. Swartz and M. Skobe, "Lymphatic function, lymphangiogenesis, and cancer metastasis," Microsc. Res. Tech. 55(2), 92-99 (2001).

3. K. E. Volmar et al., "Turnaround time for large or complex specimens in surgical pathology: a College of American Pathologists Q-Probes study of 56 institutions," Arch. Pathol. Lab. Med. 139(2), 171-177 (2015).

4. D. L. Weaver, "Pathology evaluation of sentinel lymph nodes in breast cancer: protocol recommendations and rationale," Mod. Pathol. 23(Suppl. 2), S26-S32 (2010).

5. C. H. van Deurzen et al., "Predictive value of tumor load in breast cancer sentinel lymph nodes for second echelon lymph node metastases," Cell Oncol. 29(6), 497-505 (2007).

6. S. Zahoor et al., "Sentinel lymph node biopsy in breast cancer: a clinical review and update," J. Breast Cancer 20(3), 217-227 (2017).
7. D. N. Krag et al., "Sentinel-lymph-node resection compared with conventional axillary-lymph-node dissection in clinically node-negative patients with breast cancer: overall survival findings from the NSABP B-32 randomised phase 3 trial," Lancet Oncol. 11(10), 927-933 (2010).

8. A. E. Giuliano et al., "Association of occult metastases in sentinel lymph nodes and bone marrow with survival among women with early-stage invasive breast cancer," JAMA 306(4), 385-393 (2011).

9. A. Kokolakis et al., "Prehistological evaluation of benign and malignant pigmented skin lesions with optical computed tomography," J. Biomed. Opt. 17(6), 066004 (2012).

10. A. U. Eriksson et al., "Near infrared optical projection tomography for assessments of $\beta$-cell mass distribution in diabetes research," J. Vis. Exp. 71, e50238 (2013).

11. M. Oldham et al., "Improving the quantitative accuracy of optical-emission computed tomography by incorporating an attenuation correction: application to HIF1 imaging," Phys. Med. Biol. 53(19), 5371-5383 (2008).

12. J. Sharpe et al., "Optical projection tomography as a tool for 3D microscopy and gene expression studies," Science 296(5567), 541-545 (2002).

13. J. McGinty et al., "In vivo fluorescence lifetime optical projection tomography," Biomed. Opt. Exp. 2(5), 1340-1350 (2011).

14. N. Andrews et al., "Visualising apoptosis in live zebrafish using fluorescence lifetime imaging with optical projection tomography to map FRET biosensor activity in space and time," J. Biophotonics 9(4), 414-424 (2016)

15. S. P. X. Davis et al., "Slice-illuminated optical projection tomography," Opt. Lett. 43(22), 5555-5558 (2018).

16. J. Mayer et al., "OPTiSPIM: integrating optical projection tomography in light sheet microscopy extends specimen characterization to nonfluorescent contrasts," Opt. Lett. 39(4), 1053-1056 (2014).

17. A. Bassi et al., "Optical tomography complements light sheet microscopy for in toto imaging of zebrafish development," Development 142, 1016-1020 (2015).

18. V. Kumar et al., "Optical projection tomography reveals dynamics of HEV growth after immunization with protein plus CFA and features shared with HEVs in acute autoinflammatory lymphadenopathy," Front. Immunol. 3, 282 (2012).

19. L. Quintana and J. Sharpe, "Preparation of mouse embryos for optical projection tomography imaging," Cold Spring Harb. Protoc. 2011, 664-669 (2011).

20. C. Vinegoni et al., "In vivo imaging of Drosophila melanogaster pupae with mesoscopic fluorescence tomography," Nat. Methods. 5(1), 45-47 (2008).

21. M. S. Ozturk et al., "Mesoscopic fluorescence tomography of a photosensitizer (HPPH) 3D biodistribution in skin cancer," Acad. Radiol. 21(2), 271-280 (2014).

22. Q. Tang et al., "High-dynamic-range fluorescence laminar optical tomography (HDR-FLOT)," Biomed. Opt. Express 8(4), 2124-2137 (2017).

23. M. J. Niedre et al., "Early photon tomography allows fluorescence detection of lung carcinomas and disease progression in mice in vivo," Proc. Natl. Acad. Sci. U. S. A. 105(49), 19126-19131 (2008).

24. L. Fieramonti et al., "Time-gated optical projection tomography allows visualization of adult zebrafish internal structures," PloS one 7(11), e50744 (2012).

25. F. Vasefi et al., "Angular domain transillumination imaging optimization with an ultrafast gated camera," J. Biomed. Opt. 15(6), 061710 (2010).

26. L. Sinha et al., "Comparison of time- and angular-domain scatter rejection in mesoscopic optical projection tomography: a simulation study," Biomed. Opt. Express 10(2), 747-760 (2019).

27. V. C. Torres et al., "Excised whole lymph node imaging for cancer staging with angular restriction dual fluorescent optical projection tomography," in IEEE 16th Int. Symp. Biomed. Imaging (2019).

28. K. M. Tichauer et al., "Microscopic lymph node tumor burden quantified by macroscopic dual-tracer molecular imaging," Nat. Med. 20(11), 1348-1353 (2014).

29. A. N. Bashkatov, "Optical properties of skin, subcutaneous, and muscle tissues: a review," J. Innov. Opt. Health Sci. 4, 9-38 (2011).

30. S. L. Jacques, "Optical properties of biological tissues: a review," Phys. Med. Biol. 58, R37-R61 (2013).

31. A. C. Croce and G. Bottiroli, "Autofluorescence spectroscopy and imaging: a tool for biomedical research and diagnosis," Eur. J. Histochem. 58(4), 2461 (2014). 